\title{
Une coexistence créative entre théologie et sciences des religions: quel travail à déployer, des deux côtés?
}

\section{Creative coexistence between theology and the sciences of religion: what efforts should be made on both sides?}

Pierre Gisel *

\begin{abstract}
Résumé
Ce texte sanctionne la double existence de la théologie d'une part, des sciences des religions de l'autre. Cette dualité est non dépassable, pour des raisons de fond relatives aux " intérêts " humains et sociaux en cause à chaque fois. Mais cette dualité peut être féconde pour chacune. Moyennant bien sûr qu'on les repense, l'une comme l'autre. Ce à quoi s'exerce la présente contribution. Qui précise au final en quoi la théologie, ainsi repensée, peut être riche d'enseignements pour les sciences des des religions, et en quoi les sciences des religions sont pleines de richesses humaines instructives pour le travail et la réflexion théologiques, tout en étant en même temps un lieu de défis et une occasion de déplacements qui ne peuvent qu'en relancer la réflexion.
\end{abstract}

Mots-clés: Le théologique; sciences des religions; critique; dialogue; coexistence créative.

\begin{abstract}
This text endorses the dual existence of theology, on the one hand, and religious studies, on the other. Due to substan ve reasons related to human and social «interests» called into ques on simultaneously, this duality cannot be overcome. Yet it can be bene cial to both. As long as both of them are reconsidered, however. That is the scope of the present essay. It speci es how theology, thus reinterpreted, can be highly enlightening for religious studies and how religious studies are full of human wealth directly instruc ve for theological work and thought, while at the same me posing a challenges and an opportunity for change that can only revitalize re ec on on the topic.
\end{abstract}

Keywords: The theological; religious studies; critical; dialogue; creative coexistence.

Article reçu le 5 mai 2018 et approuvé le 7 juin 2018.

* Docteur en Théologie. Professeur titulaire à l'Université de Lausanne (Suisse) de 1976 à 2012. Depuis 2012, il est Professeur Honoraire de sa Faculté de Théologie et de Sciences des Religions. Pays d’origine: Suisse. Email: pierre.gisel@unil.ch

Horizonte, Belo Horizonte, v. 16, n. 50, p. 729-749, maio/ago. 2018 - ISSN 2175-5841 


\section{Propos introductif}

Les sciences des religions - sous l'appellation de « sciences religieuses » en France ou de « Religionswissenschaft » en Allemagne - se sont développées dans la dernière partie du XIXe siècle. Elles sont ainsi inscrites dans un contexte d'émancipation, voire de laïcisation. On a dès lors souvent considéré que les sciences des religions devaient remplacer la théologie. Ou, tout au moins, qu'elles seules étaient légitimes en rationalité publique et donc en Université d’État, la théologie n'ayant du coup sa place que dans des Facultés ou autres institutions dépendant des Églises et selon une finalité liée aux Églises.

Par rapport à cette manière de voir et de faire, vieille de plus d'un siècle mais toujours présente - et même spontanément la plus fréquente -, le texte qui suit tente d'opérer un déplacement. Il ne vise ni à substituer une des deux approches à l'autre, ni à réduire la différence qu'elles cristallisent, chacune pour sa part. Il vise au contraire, d'abord, à ce que l'on se mette bien au clair touchant ce qui est engagé sur chacune des deux approches ou types d'approche. Et il esquisse, ensuite, une perspective tentant de montrer comment, chacune pour sa part, la théologie comme les sciences des religions, peut à la fois faire valoir sa spécificité - mais alors repensée hors des idéologisations qui les guettent, des deux côtés, même si c'est différemment - et tirer un profit, chacune pour sa part et selon sa fonction propre, d'être entrée sur le terrain de travail de l'autre.

\section{La perspective ouverte}

Mon titre dit un objectif ${ }^{1}$ : une coexistence possible, désirable même et créative pour chacun des deux côtés en présence.

Mon titre sanctionne aussi la double existence, de la théologie d'un côté, de sciences des religions de l'autre. Il n'annonce pas une fusion des disciplines, ni une

\footnotetext{
${ }^{1}$ La présente contribution reprend, revu et un peu amplifié, un exposé donné à Trento le 3 juillet 2015 (40e anniversaire du Centro per la Scienze Religiose, Fondazione Bruno Kessler), exposé paru dans les Annali di studi religiosi (GISEL, 2016c) et repris en anglais, à l'invitation expresse d'Alberto Melloni, lors du congrès inaugural de l'European Academy of Religion 2017, à Bologna, le 18 juin 2017.
}

Horizonte, Belo Horizonte, v. 16, n. 50, p. 729-749, maio/ago. 2018 - ISSN 2175-5841 
homogénéisation de l'espace des questions, ni non plus une transformation de la théologie en sciences des religions (voire une subordination des sciences des religions à une théologie, le modèle courant de cette dernière dût-il alors se modifier).

\section{Un arrière-plan conflictuel, dont prendre acte et à différencier}

Que l'arrière-plan des rapports entre théologie, d'une part, sciences des religions, de l'autre, soit le plus souvent conflictuel, chacun le sait. S'y tiennent les questions de la laïcité, de la sécularisation, de la «sortie de la religion » (Marcel Gauchet), avec les conflits entre la République et l'Église - conflits emblématiques en France -, à quoi s'ajoute où s'entremêle la critique de la religion, qu'elle soit menée au nom de la science (dès le temps des Lumières), qu'elle soit politique (de la Priestertrugtheorie à Marx et au-delà), psychologique (de Freud à la psychologie cognitive) ou culturelle (Nietzsche et bien d'autres). Les organisations académiques de l'étude et de la recherche sur le champ religieux en sont marquées².

En termes de conflits récurrents, diversement présents selon les lieux et moments, on peut distinguer, me semble-t-il, plusieurs ordres de débats.

\section{a) Neutralité ou engagement}

Une première opposition peut se faire jour, entre la neutralité scientifique qui serait celle des sciences des religions et le caractère engagé, confessant, de la théologie, cette dernière étant en outre insérée dans des unités académiques liées à l'Église, alimentant dès lors le soupçon - ou au contraire, selon les cas, une validation spécifique - que les théologiens y seraient des «intellectuels

\footnotetext{
${ }^{2}$ La Faculté qui fut la mienne (elle a passé du statut de Faculté de théologie protestante à celui de Faculté de sciences des religions, mais selon un processus non pleinement abouti, la Faculté peinant à se déployer en termes de pertinence sociale et ayant connu quelques retours qui montrent que des enjeux étaient restés insuffisamment clarifiés) a été le lieu d'une dispute qui vaut exemple et qui, par-delà les conflits institutionnels ou entre tel et tel acteur, a été traversée d'une foule de questions de fond instructives; cf. à ce propos mon petit livre: Gisel (2011b).
}

Horizonte, Belo Horizonte, v. 16, n. 50, p. 729-749, maio/ago. 2018 - ISSN 2175-5841 
organiques » (Antonio Gramsci), faisant passer les intérêts de leur institution ou de leur militance avant le respect de la vérité et l'honnêteté du débat.

Sur cet arrière-plan, plusieurs entendent aujourd'hui mettre en cause ce qui passerait naïvement pour objectivité scientifique, en convoquant à des fins relativistes une sociologie ou une histoire des sciences. Il me paraît personnellement que les conclusions alors tirées sont souvent trop radicales et insuffisamment différenciées : la question de ce qu'il faut ou de ce qu'on peut entendre sous objectivité scientifique doit certainement être retravaillée et approfondie, mais on devra le faire hors une disqualification avancée au seul prétexte que personne n'est neutre ou que les évidences changent selon l'histoire, voire selon les aires de civilisation.

En parallèle, plusieurs entendent aujourd'hui revaloriser les droits de la conviction et lui faire une place, mais, là encore, si le point doit effectivement être repris et travaillé, cela doit se faire, à mon sens, selon diverses précisions, distinctions de plans et de pertinences et, là encore, hors toute conclusion radicalisante.

On peut ajouter une note de psychologie des mentalités : à l'époque moderne, en l'occurrence la seconde partie du XIXe siècle, les sciences des religions sont souvent nées au sein de Facultés de théologie protestantes (allemandes, néerlandaises, suisses) et ont vécu ou vivent encore leur développement comme un processus d'émancipation progressive, non toujours terminé à leurs yeux. D'où une vigilance soupçonneuse, ainsi que la récurrence d'affirmations avançant qu'elles sont foncièrement autres que la théologie, leur constitution même réclamant la nécessité de s'en démarquer.

\section{b) Pluralité religieuse ou poursuite d'une tradition déterminée}

Une deuxième opposition se donne à voir autour de la présence et de la prise de conscience d'une pluralité religieuse, que les sciences des religions honoreraient nativement, alors que la théologie couvrirait l'ensemble du champ de sa prétention à le penser en dernière instance, voire à le réguler, sur fond d'une tradition unique, 
fût-elle intérieurement diversifiée et eût-elle connu mutations ou discontinuités historiques.

Mais, notons-le déjà, l'avènement des sciences des religions est porteur de plus que d'une attention à la pluralité religieuse. Dès lors, l'opposition entre sciences des religions et théologie - ou son dépassement en vue d'une coexistence pacifique - ne peut se résoudre en mettant en place des plateformes académiques Facultés ou autres - pluriconfessionnelles ou en intégrant à leur travail la pluriconfessionnalité ou la diversité des traditions (le judaïsme, l’islam, l'hindouisme, le bouddhisme, le taoïsme, le confucianisme, d'autres encore, à côté du christianisme), même si un dialogue, de l'échange, voire de l'interaction, peuvent être fructueusement développés sur un tel arrière-plan.

\section{c) Un décalage ou un décentrement des données et des interrogations}

Par rapport au champ dans lequel évolue la théologie, il convient de bien mesurer - cela ouvre un troisième ordre de débats - que les sciences des religions opèrent un décalage ou un décentrement. Non seulement parce que leurs types d'interrogation sont différents et non homologues, mais parce que le religieux ne constitue plus, à leurs yeux, un objet ou un champ qui serait toujours le même, se donnant à observer sur un terrain qu'on pourrait baliser quels que soient les moments historiques ou les aires de civilisation, seules la manière de remplir les cases dessinées sur ce terrain ou les manières d'y jouer pouvant différer (la case Dieu ou référence de dernière instance; les cases médiation, symbolisation, ritualité, communauté ou Église, moment constitutif ou originaire, régulation, etc.).

Pour commencer, il convient de rappeler ici que toutes les formes religieuses ne développent pas de théologie, et que cela n'est pas circonstanciel, mais touche à des types religieux différents. Par delà, il convient d'enregistrer que ce qu'on entend par religion est en débat, avec notamment la question de savoir si religion entraîne, ou non, organisation communautaire ou ecclésiale propre. Voir, à ce propos, ce qu'évoquent les deux références majeures en la matière dans notre histoire, le relegere d'une part (une vertu humaine de retrait devant la démesure 
du cosmos et de relecture des signes qui s'y donnent à déchiffrer, une vertu opposée à l'hubris), que ressaisit Cicéron à la fin de l'Antiquité préchrétienne, le religare d'autre part (relier, verticalement, à une transcendance, et horizontalement pour constituer une communauté ou de la socialité), de fait progressivement privilégié en christianisme et en Occident 3 , pour être finalement seul mis en avant avec les Temps modernes4, hors ésotérismes. Voir aussi la question de savoir ce qu'il en est de la distinction, ou non, de la religion à l'endroit $\mathrm{du}$ culturel (en Inde ancienne, fonds culturel et fonds religieux sont indissociables, de même que coïncident le symbolique et le religieux dans les sociétés premières explorées par les anthropologues5) ou à l'endroit du politique (le théologicopolitique a une histoire circonscrite, aussi bien quant à son émergence que quant à son destin).

\section{Le geste théologique rendu à une particularité donnée, et en outre soumis à procès critique}

Parmi les diverses sciences des religions, l'anthropologie, née de la rencontre d'autres systèmes culturels (suite aux découvertes modernes de l'Asie, de l'Amérique latine, de l'Océanie, de l'Afrique), a souligné combien les cultures non chrétiennes et hors monothéismes vivaient d'abord de procédures de négociation avec le monde et ce qui y échappe (l'étrangeté, la mort, les frontières, l'altérité, etc.), de la négociation se répétant dans du pratico-rituel6. On est là dans une autre perspective que celle d'un «ordre du sens», que la religion soutiendrait et nourrirait.

\footnotetext{
${ }^{3}$ Mais THOMAS D'AQUIN par exemple, dans sa Somme de théologie, de 1266-1273, Ila llae, Q. 81-100, développe un petit traité de la religion qui reprend en tout point la perspective de Cicéron (le religare avait au contraire été privilégié par Lactance ou Saint Augustin notamment).

${ }^{4}$ Émile Durkheim (1912) le sanctionne, dans Les Formes élémentaires de la vie religieuse, de 1912, à la fois quand il définit la religion comme " système de croyances » et quand il avance qu'il n'y a pas de religion sans « Église ", ce qui est à la fois typique et faux.

${ }^{5}$ Notons que le religieux, ici, ne connait pas de distinction entre du canonique et du non-canonique, et que cette distinction accompagne probablement un religieux séparé et, en tant que tel, lié à hétérogénéité ou à transcendance.

${ }^{6}$ Pour exemple, Silvia Mancini (2014, p. 93-110).
} 
La théologie, quelle qu'elle soit, apparaît alors justement, en contraste, liée à la mise en place d'un " ordre du sens », avec son organisation en système, investi d'intelligibilité humaine (la question de Dieu, de sa mort ou de ses substituts y étant en outre de fait centrale, en termes d'instance dernière), et avec sa thématique d'une adhésion subjective, un «croire », requis implicitement ou sur mode de convocation à assumer (moment ou un sujet répond de ce qui est en jeu).

Il y a donc du religieux ou supposé tel dans des mondes où la question de Dieu (quelles que soient ses variantes) ne se pose pas et où n'est pas non plus engagé un croire (là aussi : quelles que soient ses variantes). Du coup, la théologie, dans ce qui fait son geste ou sa posture, apparait liée à la particularité d'une manière de se situer dans le monde et d'y envisager l'humain. Renvoyant à transcendance, elle est alors, au mieux, une manière parmi d'autres de répondre de ce qui, au cœur même du monde, est en excès de l'humain ou le déborde, et cette manière peut être soumise à critique. Elle est de fait aujourd'hui critiquée comme anthropocentrisme, réduction à l'humain et à ses appropriations, et comme primat impérialiste accordé à la raison et à l'intelligibilité.

La critique se fait d'autant plus insistante que notre temps - postmoderne semble sortir de l'orbite indiquée, marquée par un renvoi à de la transcendance et par la venue à l'avant-scène d'un sujet assigné à répondre de lui-même et du monde7. On assiste en effet aujourd'hui à diverses propositions de "spiritualité sans Dieu » ou de «spiritualité laïque » (on dit alors significativement "spiritualité " plutôt que "religion ») ${ }^{8}$, sur un arrière-fond renvoyant à des équilibres de vie et à de la sagesse ainsi qu'aux déploiements d'un « développement personnel », plus branchés sur des «énergies cosmiques » qu'en lien à de la transcendance, autre, contestatrice et revendicatrice.

Par-delà ces indications venant de l'anthropologie et par-delà ces déplacements à l'œuvre au cœur du contemporain, c'est la définition même de ce qu'il faut entendre par religion qui se fait poreuse ou se défait. Ce que j’ai ailleurs

\footnotetext{
${ }^{7}$ Temps d'une posture de type " hérö̈que », comme le signale bien Charles Taylor (2011), liée à « mobilisation », dirait Peter Sloterdijk (2000).

${ }^{8}$ Cf. André Comte-Sponville (2006); Luc Ferry (2010); Ronald Dworkin (2014). Voir aussi ce qu'on nomme, dans les pays anglo-saxons, les « Spiritual But Not Religious » (SBNR); cf., entre autres références, Robert C. Fuller (2001).
}

Horizonte, Belo Horizonte, v. 16, n. 50, p. 729-749, maio/ago. 2018 - ISSN 2175-5841 
appelé du «religieux diffus» (les mouvances du type New Age par exemple) l'attestait déjà̀.

On est du coup conduit à entrer dans une perspective qui ne se centre pas sur le religieux, quelle que soit la diversité de ses manifestations, mais le décale et l'inscrit dans un ensemble plus large, celui de « dispositifs » qui balisent le socioculturel et commandent au statut et à la fonction du religieux, des « dispositifs » historiquement changeants ${ }^{10}$. C'est là que se tient la raison de fond qui empêche qu'on se contente d'organiser le travail simplement en fonction de plusieurs traditions (une pluriconfessionnalité) et qui empêche aussi que soit construit un espace intellectuel de questions qui seraient en tant que telles religieuses et en fonction desquelles on travaillerait les diverses cristallisations religieuses aussi bien que leurs recompositions.

Concrètement, il y a au contraire à entrer dans une perspective qui soit organisée en fonction d'une scène religieuse - une scène changeante - sur laquelle viennent se dire du social et de l'anthropologique, dont elle est un lieu de symptômes ${ }^{11}$.

On articulera dès lors l'étude du religieux - quel qu'il soit - à la société civile et aux pluralités qui la traversent, société alors vue comme un tiers, à quoi s'ajoutera une considération touchant plus spécifiquement le politique, donc l'État et sa laïcité (l'État à la fois comme arbitre et soucieux de favoriser l'expression des différences internes au civil, à l'encontre de toute homogénéisation et unidimensionnalité). La société civile est ici un tiers requis, empêchant tant une focalisation sur le seul religieux qu'un face-à-face entre un religieux et ce qui pourrait en être un substitut de même niveau et de même fonction (telle forme de l'État, voire telle organisation de la société civile).

\footnotetext{
${ }^{9}$ Cf. mon petit livre: Gisel (2007); et la traduction italienne, légèrement revue: Gisel (2011a).

${ }^{10}$ Le mot dispositif renvoie à Michel Foucault (cf. Paul Veyne, 2008), que reprend Giorgio Agamben (2007).

${ }^{11}$ Pour plus, je renvoie à ma contribution: Gisel (2016b); original français repris et adapté: Gisel (2016e); et traduction italienne: Gisel (2017a).
}

Horizonte, Belo Horizonte, v. 16, n. 50, p. 729-749, maio/ago. 2018 - ISSN 2175-5841 


\section{Pour une mise en perspective généalogique des questions, liée à une construction de problématiques}

Ce sur quoi ouvre le point précédent l'anticipait : en matière de travail sur le religieux et de mises en avant des enjeux, il y a lieu d'entrer dans une perspective de longue durée et comprenant une comparaison d'aires de civilisation. Les dispositifs qui commandent au religieux ou à ce qu'on peut appeler tel ne sont en effet pas les mêmes dans l'Antiquité gréco-romaine (cf. par exemple les travaux de Jean-Pierre Vernant, Marcel Detienne ou John Sheid) que dans ce qui a fait la tradition majoritaire de l'Occident, ni ne sont les mêmes en Inde ou en Chine par exemple.

On inscrira dans cette mise en perspective de longue durée et comparative une lecture généalogique de notre présent - sur laquelle seront greffées des problématiques à élaborer -, qui fera voir des déploiements divers permettant de profiler des enjeux et, partant, d'esquisser des typologies. Parmi ces questions, à la fois centralement en cause dans le «D'où venons-nous? " et le «En quoi en sortons-nous et pour quoi ? (qui ouvre du coup un questionnement sur l'aujourd'hui), on peut évoquer : a) ce qui est à entendre sous religion; b) la question de Dieu, d'une transcendance ou de ce qui se tient en excès ; c) ce qu'il en est de la posture du croire et, liée, la question du sujet ${ }^{12}$; d) le rapport au monde dans ce qu'il a de non réductible à humain (classiquement dit, le cosmos), entre anthropocentrisme et reconnaissance tant des limites que de ce qui fait les aléas et l'épaisseur du réel; e) le «récit » qu'on se donne explicitement ou non de la modernité ${ }^{13}$, où se nouent identité et projet possible, trop souvent unilatéralement mise en avant comme émancipation progressive ou, à l'inverse, investie de nostalgie réactive ou, encore, traversée d'utopique, de formes variées. Avec, à chaque fois, la diversité interne à chacune de ces thématiques et leurs avatars.

\footnotetext{
${ }^{12}$ Sur les questions, couplées, de Dieu et du sujet, Slavoj Zizek me parait ici typique, Peter Sloterdijk également.

${ }^{13} \mathrm{Cf}$. ce que Jean-François Lyotard (1979) appelle les " grand récit »; au plan individuel, le « récit de soi » est validé chez Paul Ricœur, en lien à la vision d'une « identité narrative » (cf. aussi Judith Butler, 2007), ainsi, bien sûr, que dans les programmes de " développement personnel » et analogues.
}

Horizonte, Belo Horizonte, v. 16, n. 50, p. 729-749, maio/ago. 2018 - ISSN 2175-5841 
A mon sens, une telle mise en perspective généalogique, avec la problématologie qui l'accompagne (construite sur le fond d'une intrigue touchant l'avènement de l'humain, donc de pulsions ou de désirs à faire apparaître et à valider, articulés à des motifs visés, l'ensemble étant mis en place à partir d'un présent et des problèmes ou apories qui s'y tapissent, à déchiffrer et à diagnostiquer là aussi), est requise qu'on soit en théologie, en sciences des religions ou en philosophie de la culture et du social.

Mettre en place la perspective généalogique indiquée suppose qu'on s'articule au socio-culturel comme tel, non à telle ou telle tradition. Cela dit, les traditions ont donné et donnent forme à ce qui est en jeu dans chacune des problématiques sur lesquelles on va inscrire le travail et la réflexion. Et c'est là une donne non évacuable, aussi vrai qu'il n’y a de prises en charge de ces questions que sous des formes particulières et historiquement situées, des prises en charge qui en répondent, passant du coup par une adhésion propre et une manière de construire une identité, par-delà des discontinuités effectives.

On est dès lors conduit à mettre en place deux modèles de travail, selon une compatibilité à penser, mais hors homogénéisation. Et, institutionnellement, on ne répondra pas du religieux sans que se pensent ou se repensent et se mettent en place des Facultés ou autres types de plateformes académiques organisées en fonction d'une tradition, d'une part, des Facultés ou autres type de plateformes académiques organisées en fonction de la scène religieuse, d'autre part, où se tiennent plusieurs traditions, mais d'autres choses aussi que des traditions, et où se jouent divers rapports à la société comme telle. Les premières intègrent à leur travail la question de savoir quelles formes elles doivent et peuvent donner à leur tradition dans un présent ou un contexte neuf : ce ne sont pas les unités organisées en fonction de la scène religieuse globale qui diront ce que doit et peut être le christianisme ou l'islam aujourd'hui et en tel lieu, bien que ce qui s'y fait puisse être indirectement utile à une réflexion portée par et portant une tradition. Mais, tout en étant articulées à une tradition, ces Facultés ou unités académiques seront en même temps utiles à la société : il est en effet bon pour tous, indirectement, qu'une tradition travaille sur son histoire et son présent. Ainsi, si cela fait indéniablement 
partie de la responsabilité intellectuelle globale de la société de nourrir des unités d'enseignement et de recherche vouées aux questions religieuses (ces questions sont de pertinence et d'effets sociaux), sans les laisser aux seules traditions qui les portent (il y a lieu de les examiner de manière décentrée), il est aussi utile, pour tous, qu'il y ait des Facultés islamiques, chrétiennes et autres, et il peut même être adéquat d'en favoriser le déploiement ${ }^{14}$.

\section{Pour une coexistence créative entre théologie et sciences des religions}

Les sciences des religions sont riches de tout un savoir sur l'humain dans ses rapports au monde, à lui-même et à ce qui le dépasse, cristallisés au gré d'une très grande variété de dispositifs et d'agencements commandant à des habitations de l'espace et du temps, à des symbolisations du social, à de la ritualité, à des mises en place de références, de mémoires et de traditions, aux manières de concevoir les jeux institutionnels, avec leurs instances et pouvoirs divers, leurs modifications ainsi que les moments de dissidences et d'utopie qui les traversent, à des imaginaires aussi, qui orientent, balisent, ouvrent et conditionnent la vie.

Les sciences des religions sont diverses. Il n'y a pas une science des religions, mais un faisceau de disciplines différentes et mises ici au travail - de l'histoire, de l'anthropologie, de la sociologie, de la psychologie -, et on pourrait y mobiliser, plus articulés à du régulatif (mais le religieux en est chargé), du droit, de la politologie, de la philosophie, du médical, d'autres approches encore. Non une discipline donc, mais un faisceau de disciplines, en fin de compte parce que la religion ou le religieux ne constitue pas à proprement parler un objet, commandant une méthode d'approche et de traitement. Ce qu'il en est du religieux est au contraire une question ouverte, dont on ne tranche pas hors d'enjeux ni, du coup, hors d'interrogations transversales. Tout au plus, mais ce n'est pas rien, y a-t-il une histoire du traitement des religions (en modernité, depuis environ 150 ans), et cette histoire fait voir un ensemble de problèmes qui, comme tels, circonscrivent

\footnotetext{
${ }^{14}$ Sur cet ensemble, cf. ma contribution: Gisel (2013b).
}

Horizonte, Belo Horizonte, v. 16, n. 50, p. 729-749, maio/ago. 2018 - ISSN 2175-5841 
bien un champ de travail, y compris le fait que ces problèmes y ont été posés, abordés et réfléchis de manière diverse, contradictoire, voire polémique.

Notons qu'il en va de même en théologie, elle aussi aujourd'hui articulée à des savoirs divers, historiques et sociaux, ce qui est proprement théologique étant transversal à ce qui se déploie et s'observe sur chacun des champs travaillés (concrètement: des savoirs sur la Bible et ses contextes, sur l'histoire du christianisme, avec ses diverses productions, doctrines comprises, sur les données du présent et ce qui s'y passe, etc.) et étant en ce sens second: foncièrement problématisant et réflexif. Et là aussi se donne l'histoire d'un déploiement diversifié, celui de la théologie, y compris pour ce qui touche son statut, une histoire instructive en tant que telle et quant à ce qui y est en jeu.

Les religions sont diverses et particulières à chaque fois. Même leurs prétentions à l'universel - quand elles en ont, ce qui n'est pas toujours le cas - sont déterminées par une perspective particulière. Et, sauf à en sanctionner une comme seule vraie, et vraie dans sa positivité même (ce qu'elle peut être tentée de faire, mais alors pour sa perte, devenant pure idéologisation et idolâtrie), une théologie ne dépassera pas ces particularités. Tout au plus, mais c'est décisif, pourra-t-elle les rendre fructueuses ${ }^{15}$, au gré de leur historicisation et des déplacements et confrontations qui peuvent en découler.

Sauf l'illusion d'une raison universaliste (qui a pu prendre, en modernité, la forme d'une vision progressiste, de l'animisme au polythéisme, puis au monothéisme, pouvant se prolonger en direction de la métaphysique et, enfin, de la science), les sciences des religions n'ont pas non plus de quoi dépasser la diversité et les particularités des religions, sauf à croire qu'elles peuvent circonscrire leur objet, le religieux (alors que c'est un champ de questions, certes déployées en des positivités examinables), et en proposer un savoir (il y en a des savoirs, partiels, liés à des points de vue divers et chacun en principe pertinent).

\footnotetext{
${ }^{15}$ Pour un développement, cf. ma leçon d'adieu: Gisel (2014b), traduction italienne: Gisel (2013a); cf. aussi mon exposé donné à Paris le 7 novembre 2017, "Rendre fructueuses pour tous les particularités héritées. Selon quelles conditions et quel type de relecture ? " (colloque Le désir d'universalité, Institut de France et École Normale Supérieure).
}

Horizonte, Belo Horizonte, v. 16, n. 50, p. 729-749, maio/ago. 2018 - ISSN 2175-5841 
C'est non sans lien avec cette donne que le religieux est traversé de traditions, avec la tentation, chez les uns (c'est le cas chez certains théologiens), d'en sanctionner une comme bonne, à moins de proposer qu'on travaille et habite chacune en juxtaposition, sur fond de tolérance, mais sans autre profit, et avec la difficulté, chez les autres (du côté des sciences des religions), à en penser le fait. Au prétexte que les traditions sont des ripostes construisant une identité par-delà des discontinuités effectives (ce qui est indéniable), on en laisse en effet volontiers de côté la donne sans en proposer une intelligence propre, abandonnant à l'idéologique le fait de cette construction, pourtant humainement et socialement central, et restant du coup impuissant à penser tant les basculements historiques que ce qui y naît et s'y noue à chaque fois. On ne travaillera ni ne pensera plus, par exemple, le christianisme ou l'islam, mais des christianismes ou des islams, chaque fois rapportés à tel moment et à tel lieu, toute autre position ne pouvant, pense-ton ici, que conduire à « essentialisme »16.

\section{a) Retour sur la théologie}

Passer par les sciences des religions et ce qu'elles font voir est de grand profit pour le travail théologique, et d'abord parce qu'il s'y trouve inscrit sous un horizon plus large et dès lors avec la possibilité de penser quel type de religion est la tradition - par hypothèse, le christianisme - dont la théologie entend répondre, avec ses forces et ses risques propres, liés à la manière qui est la sienne de donner forme particulière à des dispositions humaines générales et ouvertes.

Plus largement, entrer sur le terrain travaillé par les sciences des religions permet à la théologie de déployer une part de ce qu'il est convenu d'appeler, en vocabulaire catholique et depuis le milieu du XXe siècle, une «théologie fondamentale », distincte de la «dogmatique ». Le terrain en cause représente alors, formellement, un analogue à ce qu'ont pu par exemple offrir, au XIIIe siècle, la métaphysique ou, au XVIIe, les théologies philosophiques ayant développé des

\footnotetext{
${ }^{16}$ J'ai repris cette thématique dans Gisel (2017b); traduction italienne: Gisel (2018).
} 
théodicées, ou encore, aux XIXe et XXe siècles, une théorie transcendantale du sujet ${ }^{17}$.

$\mathrm{Au}$ plan des données spécifiques à la tradition considérée (relevant de la « dogmatique » plus que de la «théologie fondamentale»), un passage par les sciences des religions permettra qu'on en examine et qu'on en pense chacune des propositions concrètes, symboliques, rituelles, doctrinales, institutionnelles et autres, en y faisant résonner les réalités humaines dont, de fait, elles répondent à chaque fois. Aucune de ces propositions ne vaut en effet pour elle-même, comme un « bien de salut » renvoyant à extrinsécisme, et du coup non sans réseau autoréférencié. Prétendre le contraire serait se condamner à ne pas pouvoir donner une intelligence de ces propositions et supposerait en outre un système religieux et du croire littéralement aliénants. Toute affirmation et mise en place de données croyantes est une manière de répondre de questions humaines plus larges et en tranchant d'enjeux se situant à ce niveau-là ${ }^{18}$; les comprendre suppose dès lors de les examiner sous ce regard, et sur le fond d'une pluralité permettant de les profiler et de les évaluer.

En tout cela se nourrit, pour le travail et la réflexion mêmes de la théologie, une créativité liée à une fréquentation des sciences des religions, dont la théologie va faire son profit à l’interne.

\section{b) Retour sur les sciences des religions}

Le profit d'une rencontre ou de croisements avec la théologie est, pour les sciences des religions, indirect, mais non marginal, ni facultatif. Comme le profit d'un travail sur le terrain des sciences des religions était, pour la théologie, indirect, tout en touchant au cœur de sa tâche.

La théologie est liée à une tradition, y assurant même, entre autres choses, une part de sa régulation, avec ce qui s'y tient de ripostes aux défis du temps, avec

\footnotetext{
${ }^{17}$ Cf. le moment constitutif de « dépendance » chez Friedrich Schleiermacher (en lien à Kant), ou l'« existential » chez Karl Rahner ou Rudolf Bultmann (en lien à Heidegger et, différé, à Kant encore).

${ }^{18} \mathrm{Cf}$. mon livre Gisel (2012, p. 103s. e 107), de même que pour mes remarques renvoyant à une part de la tâche d'une " théologie fondamentale ».
}

Horizonte, Belo Horizonte, v. 16, n. 50, p. 729-749, maio/ago. 2018 - ISSN 2175-5841 
ses avatars aussi, tous riches d'enseignements au gré des discontinuités historiques et aujourd'hui au cœur de la sécularisation et autres recompositions sociales et religieuses. La théologie se déploie en outre comme une manière de développer du réflexif, se donnant là aussi comme objet d'observation instructif. Il n'y a en effet pas de raison que les sciences des religions prennent pour objet de travail et d'examen seulement le rite, les devenirs institutionnels, les symbolisations, et non la théologie comme telle, dans ses diverses dimensions : la théologie est partie prenante du religieux, et s'y cristallisent des questions et des données humaines, comme en toute autre réalité religieuse.

Par-delà, il me paraît qu'il y a souvent, du côté des sciences des religions, des «taches aveugles». Probablement en lien avec l'histoire moderne de leur constitution et le phénomène d'émancipation que j’avais signalé d'entrée. Ce que j'ai noté plus haut à propos du phénomène de tradition est ici typique. De même, on mettra volontiers entre parenthèses, en sciences des religions, la question du canon biblique, pour examiner tous les textes au même niveau, textes canoniques et apocryphes, une manière de faire légitime et qui représente un gain sur un certain plan, à préciser, mais qui ne doit pas conduire à un abandon pur et simple de la réflexion touchant ce qui se joue à propos et à l'occasion de cette partition : une tradition vit de canonisation - c'est au reste un phénomène humain et social qui déborde le strict religieux, même s'il se donne particulièrement à voir sur cette scène -, et la manière dont une tradition a opéré ses choix et lesquels participe de la constitution même de ce qu'elle est.

Il en va de même touchant ce qui est proprement doctrinal, là encore à ne pas exclure (ni à réserver à la seule théologie), pas plus que le fait même d'une tradition ou que la canonisation de textes de référence. En outre, que la théologie y ait déjà opéré un travail de médiatisation ou de métabolisation ne peut que faciliter - tout dût-il être traduit ou transposé - la compréhension tant de ce qui est proposé que de ce qui s'y joue et s'y noue. Pour exemple: une réflexion traditionnelle sur la «non-dualité » sera prise en compte dans la compréhension du bouddhisme, mais il devra en être de même, et au même titre, de la réflexion de la théologie chrétienne sur le «principe» (une question centrale et socio- 
culturellement largement partagée au cœur de l'Antiquité tardive) visant à en assurer un statut et une fonction spécifiques en le pensant un sans qu'il soit pour autant simple (le « principe » vaut pour la détermination et pour une effectuation, non seulement pour la provenance, ce qu'assure le penser «trinitaire » de Dieu alors engagé). Il en va de même encore - mais on pourrait allonger la liste des exemples - touchant le christologique, qui est une manière de prendre en charge la question de la médiation (une question large là aussi, et par ailleurs centrale au cœur du social contemporain et de ce qui arrive à ses institutionnalités) et de lui donner forme et statut (de l'intermédiaire, supposant en fin de compte un même espace ? du tiers ${ }^{19}$, du coup décalant et ouvrant à la métaphorisation ? autre dispositif ?).

J'ai plaidé en section 4 pour une relecture généalogique liée à la construction d'une problématologie. On se trouvait du coup articulé à un présent à différencier, dont rendre compte, voire à prendre en charge. La nécessité s'en impose finalement à tous à mon sens, au cœur même du travail à mener et de l'intelligence à conquérir, qu'on se l'explicite ou non, étant entendu qu'on peut se l'expliciter de diverses manières et à diverses fins. La théologie est de fait spontanément plus portée que les sciences des religions à entrer dans une relecture de ce type et dans les questionnements à y greffer. C'est probablement, pour commencer, que la théologie ne peut qu'être habitée par une conscience historique de longue durée et ne peut qu'être encline à mettre en cause tant des découpages en tranches successives, au surplus justement desserties d'un ensemble problématique, qu'une tendance à focaliser sur ce qui est ici seul compris comme fait - tel événement isolé, tel personnage, tel moment de production de texte, etc. , alors que ce qu'une tradition ou une société aura fait de quelque événement que ce soit, comme de quelque personnage ou de quelque texte que ce soit, ainsi que sa manière de s'y rapporter, est décisif, «faisant histoire » et relevant à ce titre de

\footnotetext{
${ }^{19}$ Sur ce statut de tiers dans ce contexte, je renvoie à ma "Réponse à Silvia Mancini ", in Gisel (2014a, 111-133), ici p. 121-124 (à l'arrière-plan se tiennent bien sûr, différement, Jacques Lacan et Emmanuel Levinas). Touchant plus largement une irréductible pertinence sociale du tiers, cf. Alain Supiot (2009).
}

Horizonte, Belo Horizonte, v. 16, n. 50, p. 729-749, maio/ago. 2018 - ISSN 2175-5841 
l'histoire justement, qui n'est pas qu'accumulation d'événements, pas plus que production de références possibles.

On aura ici compris qu'en dernière instance, il y a un plaidoyer à instruire à l'encontre d'un « trend » neutralisant inscrit au cœur des sciences des religions et participant probablement de données socio-culturelles contemporaines plus larges. Par-delà son attachement natif à la longue durée et à une problématisation, la théologie a vocation à décaler et à valider de l'hérétique (c'est moins le cas pour les sciences des religions, dans la mesure où elles entendent se déployer hors normatif et en rester au descriptif), et d'abord parce que la théologie porte la question de ce qui est hétérogène et qu'elle est amenée à montrer que de l'« hétérologique » est à l'œuvre et à en déployer les procès ${ }^{20}$. A quoi j'ajouterais volontiers que cette donne foncière - cet enjeu de fond - réclame probablement que se soient humainement et socialement cristallisées et assurées des « hétérotopies »21, en décalage et fécondité (bien comprises, quant à leur statut et à leur fonction, les communautés religieuses y ont vocation).

$\mathrm{Au}$ total, une entrée sur le terrain des objets et dans le champ réflexif de la théologie ne peut qu'être fructueuse pour les sciences des religions, même si le profit est indirect - comme je l'avais dit symétriquement pour la théologie -, toute chose devant être transposée et traduite dans leur espace propre. Cette entrée sur le terrain du théologique représente d'abord et en toute hypothèse un enrichissement en termes de données à prendre en compte, mais elle peut aussi s'avérer lourde d'une relance de questions de fond tapies au cœur même de ce qui se tisse dans le religieux et les religions, des questions humaines et sociales, à prendre en charge d'une manière ou d'une autre, ou dont tenir compte, sauf à passer à côté du matériel même à examiner et à s'exposer à revanches sourdes, même au plan intellectuel (au plan social, cela me paraît, à l'évidence, éclatant).

\footnotetext{
${ }^{20}$ On pensera ici à Michel de Certeau, dont j'ai été proche; cf. mon texte Gisel (2016d, p. 257-280).

${ }^{21}$ Le terme renvoie à Michel Foucault (2009). Reste à penser l'articulation de cet hétérotopique au social de tous et à montrer en quoi il peut y être fructueux et non donner prétexte à sectarisation; je m'y suis essayé dans Gisel (2016a) - traduction italienne: Gisel (2016f)-, ainsi que dans deux livres récents: Gisel (2017b) et Gisel; Bolli-Voélin (2017), chapitres 8 et 10.
}

Horizonte, Belo Horizonte, v. 16, n. 50, p. 729-749, maio/ago. 2018 - ISSN 2175-5841 


\section{Conclusion}

On aura compris que mon texte ne visait pas à trouver des accommodements, ni de petites inflexions permettant des rapprochements entre théologie et sciences des religions. C'est que je n'invite pas à travailler sur des frontières, à assouplir, mais à un travail interne, en lien à des enjeux. Ces enjeux doivent en outre être préalablement mis à jour, explicités et pensés, par-delà les opinions ou les représentations courantes.

Il y a donc à entrer dans une opération de déplacement - je l'avais annoncé dès mon « propos introductif » - et ce déplacement sera d'abord un décentrement, tant de chacune des disciplines ou faisceaux de disciplines en cause que de ce qui fait leur opposition même. C'est ainsi à un approfondissement qu'on est invité, comprenant une forme de critique bien sûr, mais une critique ouvrant sur un moment réflexif qui puisse reprendre, sur le fond, ce qu'il en est du statut, de la fonction et de la tâche tant de la théologie que des sciences des religions. Et où l'on voit, en fin de compte, que chacune de ces deux disciplines ou de ces deux types d'approches, dans leur différence et leur spécificité respective, a une pertinence pour tous, donc pour la société comme telle et en rationalité publique.

On aura enfin perçu que n'est pas ici à engager seulement une critique des disciplines ou es approches en cause et de leur polarisation présente. Il convient d'instruire, par-delà, un examen et un travail critiques articulés au donné socioculturel contemporain, dit postmoderne. En fin de compte, on y sera conduit à récuser une position où serait, d'un côté, sanctionné un pur multiculturalisme, fait de positions autocentrées et pouvant se déployer sur le marché libre du religieux ou des religions (c'est là la tentation qui guette aujourd'hui les traditions religieuses, le christianisme comme d'autres), et où serait, de l'autre côté, également sanctionnée une neutralisation de questionnements de fond (une propension souvent à l'œuvre au cœur des sciences religieuses, alors en phase avec un trend globalement inscrit dans le socioculturel contemporain). Ces 
questionnements sont d’importance anthropologique, sociale et humaine, mais notre temps ne sait plus les penser et même plus les énoncer. Les traditions religieuses les avaient portés (encore faut-il aujourd'hui les restituer ou les dégager de la gangue d'affirmations simplement confessionnelles), mais notre temps les estime propres aux particularismes et les leur abandonne, non sans refoulement, au gré d'une neutralisation rampante, au fond non consciente.

\section{RÉFÉRENCES}

AGAMBEN, Giorgio. Qu'est-ce qu'un dispositive? Paris: Payot \& Rivages, 2007.

BUTLER, Judith. Le récit de soi. Paris: PUF, 2007.

COMTE-SPONVILLE, André. L'esprit de l'athéisme: introduction à une spiritualité sans Dieu. Paris: Albin Michel, 2006.

DURKHEIM, Émile. Les formes élémentaires de la vie religieuse. Paris: F. Alcan, 1912.

DWORKIN, Ronald. Religion sans Dieu. Genève: Labor et Fides, 2014.

FERRY, Luc. La révolution de l'amour: pour une spiritualité laïque. Paris: Plon, 2010.

FOUCAULT, Michel. Le corps utopique; suivi de Les hétérotopies. Paris: Lignes, 2009.

FULLER, Robert C. Spiritual but not religious. Understanding Unchurched America. New York: Oxford University Press, 2001.

GISEL, Pierre. Che cosa è una religione? Brescia: Editrice Queriniana, 2011a.

GISEL, Pierre. Che cos'è una tradizione? Roma: Inschibboleth Edizioni, 2018.

GISEL, Pierre. Défis actuels: quel profil et quel service pour l'Église dans la société contemporaine? Positions Luthériennes, Paris, v. 64, n. 1, p. 59-75, 2016a.

GISEL, Pierre. Dell'universale e delle particolarità. Il Regno, Bologna, v. 58, n. 1142, p. 249-256, 2013a.

GISEL, Pierre. Du religieux, du théologique et du social: traversées et déplacements. Paris: Les Éditions du Cerf, 2012. 
GISEL, Pierre. El estatus y la función de lo religioso en la Academia como debate social. Visión desde la Universidad. Teologia y Vida, Santiago, v. 57, n. 4, p. 539-558, 2016b.

GISEL, Pierre. La coesistenza tra teologia e scienze religiose. Annali di studi religiosi, Trento, n. 17, p. 53-65, 2016c.

GISEL, Pierre. La pertinence théologique da la pensée de Michel de Certeau. L'indiscipline de l’interdisciplinarité. Teologia y Vida, Santiago, v. 57, n. 2, p. 257-280, 2016d.

GISEL, Pierre. Le religieux à l'Univesité. Didaskalia: Revista da Faculadade de Teologia de Lisboa, Coimbra, v. 46, n. 2, p. 27-46, jul. $2016 \mathrm{e}$.

GISEL, Pierre. Lo statuto e la funzione del religioso come dibattito sociale. Un punto di vista a partire dall'Università. Rassegna di Teologia, Napoli, v. 58, n. 3, p. 397-414, 2017 .

GISEL, Pierre. Qu'est-ce qu'une religion? Paris: Vrin, 2007.

GISEL, Pierre. Qu'est-ce qu'une tradition? Ce dont elle répond, son usage, sa pertinence. Paris: Hermann, 2017b.

GISEL, Pierre. Rendre fructueuses pour tous les particularités héritées: selon quelles conditions et quel type de relecture? Colloque Le désir d'universalité, Institut de France et École Normale Supérieure, 7 novembre 2017c.

GISEL, Pierre. Réponse à Silvia Mancini. In: EHRENFREUND, Jacques; GISEL, Pierre (Dir.). Mises en scène de l'humain: sciences des religions, philosophie, théologie. Paris: Beauchesne, 2014a. p. 111-133.

GISEL, Pierre. Résistances des particularités et pièges de l'universel. Pour un usage subversif des corps, des traditions et des frontières. In: EHRENFREUND, Jacques; GISEL, Pierre (Dir.). Mises en scène de l'humain: sciences des religions, philosophie, théologie. Paris: Beauchesne, 2014b. p. 227-247.

GISEL, Pierre. Sfide attuali: quale profilo e quale servizio per la Chiesa nella società contemporanea? In: ASKANI, Hans-Christoph et al. Riformare insieme la Chieza. Comunità di Bose: Qiqajon, 2016f. p. 217-240.

GISEL, Pierre. Traiter du religieux à l'université: une dispute socialement révélatrice. Lausanne: Antipodes, 2011b.

GISEL, Pierre. Une double vocation de la théologie, interne et externe. Ordres différents et compatibilité. Études théologiques et religieuses, Montpellier, v. 88, n. 3, p. 375-390, 2013b.

GISEL, Pierre; BOLLI-VOÉLIN, Michèle. L'humain entre résistance et dépassement. Entretiens sur le christianisme et le religieux en société contemporaine. Le Mont-sur-Lausanne: Ouverture, 2017. 
LYOTARD, Jean-François. La condition postmoderne: rapport sur le savoir. Paris: Minuit, 1979.

MANCINI, Silvia. Logique des fondements et logique orthopratique. Le problème théologique de la croyance à l'épreuve du culte populaire des images habillées au Mexique. In: EHRENFREUND, Jacques; GISEL, Pierre (Dir.). Mises en scène de l'humain. Sciences des religions, philosophie, théologie. Paris: Beauchesne, 2014. p. 93-110.

S. THOMAE DE AQUINO. Summa theologiae. Ottawa : Commissio Piana, 1953.

SLOTERDIJK, Peter. La mobilisation infinie. Paris: Bourgois, 2000.

SUPIOT, Alain. Homo juridicus: essai sur la fonction anthropologique du Droit. Paris: Éd. du Seuil, 2009.

TAYLOR, Charles. L’âge séculier. Paris: Seuil, 2011.

VEYNE, Paul. Michel Foucault: sa pensée, sa personne. Paris: Albin Michel, 2008. 